Results A total of 319 DRPs were identified in 235 patients, in which $280(87.8 \%)$ of 319 DRPs were deemed to be preventable. The majority of preventable DRPs were related to dose selection (219 [78\%] of 280). None of the preventable DRPs were life-threatening or fatal. The majority were assessed as moderate in severity (264 [94.3\%] of 280). There was no significant difference between DRP incidence with age (mean 3.5, $\mathrm{p}=0.389)$, sex $(\mathrm{p}=0.436)$, and weight (mean 13.47, $\mathrm{p}=0.323$ ). Younger children (aged 2 years or younger) admitted to the PICU were more likely to have a DRP (odds ratio $4.44, p=0.000)$. Scheduled admissions were 2.89 times more likely to be exposed to DRP compared with transferred admissions $(p=0.005)$. Additionally, DRP incidence increased proportionally to the number of medications.

Conclusion Our results show a high incidence of preventable DRPs, which were found to be related to dosing and drug choice problems. These results may be used for designing the epidemiology study in the pediatric population aiming to establish appropriate prevention strategies towards improvement and safe medicine use in this vulnerable patient population.

\section{COMPARISON OF THE LINKED CANCER REGISTRY AND CANCER PATIENT EXPERIENCE SURVEY DATASETS IN ENGLAND AND THE UNITED STATES}

Saleh Alessy, Margreet Lüchtenborg, Elizabeth A Davies. Cancer Epidemiology, Population and Global Health, Comprehensive Cancer Centre, Faculty of Life Sciences King's College London, UK

\subsection{6/bmjoq-2019-PSF.55}

Background Patient care experience surveys are now used internationally to assess, monitor, and improve healthcare quality. The National Cancer Patient Experience Survey (CPES) is an annual English survey that invites patients to report their experiences of National Health Service (NHS) cancer care. The Consumer Assessment of Healthcare Providers and Systems (CAHPS) survey is widely used in the USA to assess patients' experiences of healthcare plans. Several years of collated data for each survey have recently been linked to population-based cancer registry data. We aimed to compare strengths and weaknesses of the datasets in order to consider the questions they may best answer.

Methods We obtained published information about both datasets, and compared data sources, time period, patient age, cancer types, survey method and response rate, linkage method, and question themes.

Results The English dataset of 233,445 patients was created by linking 2010-2014 national CPES with cancer registry data by matching individual identifiers, whereas the US dataset of 150,750 was created by first merging national Medicare data with regional Surveillance Epidemiology with End Results (SEER) data and then with all 1998-2010 CAHPS survey data. The major differences were that the US dataset was largely limited to patients over the age of 65 years and included a large non-cancer comparison group of 571,318 patients as well as data on health costs. Both linkages included all cancer types, with breast, prostate, colorectal, and lung cancer representing $50 \%$ of English and $61 \%$ of US patients. Both were postal surveys, with non-respondents being followed up by mail in England and by telephone in the USA. Response rates were similar in England (67\%) and the USA
(71\%). The questions themes were similar, with CPES focussing on more cancer-specific experiences.

Conclusion The English dataset is likely to provide more detailed and representative data answering questions about cancer experiences in the English population. However, it may be possible to use both datasets to compare the experiences of older patients receiving government-funded cancer care in each country. The addition of economic data to English survey data as in the US data is an intriguing avenue for future research. A translated version of CAPHS is being used in Saudi Arabia, meaning that further data linkage and international comparisons may be possible in due course.

\section{ELECTRONIC OCCURRENCE VARIANCE REPORTS (EOVR) MANAGEMENT SYSTEM}

Suzette Brondial, Abdulrahman Alhasani, Mohamed Adel Elfaiomy, Mahmoud Abdelfattah Radwan. Quality Management and Planning Department, Royal Commission Health Services Program Yanbu

\subsection{6/bmjoq-2019-PSF.56}

Background The Royal Commission Health Services Program (RCHSP) has been consuming thousands of occurrence variance report (OVR) forms (paper-based) to report all hospital incidents, with 200-300 OVRs per month. This has resulted in substantial challenges for the risk management unit regarding regular tracking, trending, and analysis of all OVRs while complying to the determined timeframes. The Quality Management and Planning Department (QMPD) and information Technology (IT) Department have started collaboration on an initiative to make the incident reporting system electronic to capture incident data, do incident follow-up, and communicate incident learning across all departments through an efficient, effective, and user-friendly system with good follow-up capability. The aim of this study was to improve the timeliness, efficiency, easy accessibility, and effectiveness of the OVR system.

Methods The new system is web-based and staff can access it using their usernames and passwords. Filling an OVR is easier as data entry occurs by ticking from displayed options with the availability of entering free text for more details if needed. Automatic notifications will be sent to the relevant staff throughout the organization. A comprehensive action assignment and follow-up system has been developed. Easy access to data across the organization and updated real-time view of the OVR dashboard are also available any time. Forwarding submitted OVR(s) to appropriate entities and tracking them has become more accurate and efficient. Pilot testing has been conducted for 2 months before full implementation.

Results The electronic system has significant benefits making it more convenient to all stakeholders. The number of reported adverse events, near misses, and sentinel events further increased. The ease of access to the web-based module to fill OVRs, the real-time incident $\log$, the ability to attach any type of files to log entries, and the automatic time-stamped audit $\log /$ referencing are all features that make the new system more convenient. The action assignment and easy tracking system, the integrated emergency notification, and the electronic forms management enabled the risk management unit to manage the system more effectively and efficiently. Processing an OVR by quality staff decreased markedly from more than 120 minutes in the old system to 20 minutes in the new electronic system. The new system secured access and ability to create 